\title{
Identification of Potential Inhibitors of Calcium/Calmodulin- Dependent Protein Kinase IV from Bioactive Phytoconstituents
}

\author{
Preeti Gupta, ${ }^{1}$ Shama Khan, ${ }^{2}$ Zeynab Fakhar ${ }^{(D},{ }^{3}$ Afzal Hussain, ${ }^{4}$ Md. Tabish Rehman $\mathbb{D}^{4},{ }^{4}$ \\ Mohamed F. AlAjmi $\mathbb{D}^{4}{ }^{4}$ Asimul Islam $\mathbb{D}^{1},{ }^{1}$ Faizan Ahmad, ${ }^{1}$ and Md. Imtaiyaz Hassan $\mathbb{D}^{1}$ \\ ${ }^{1}$ Centre for Interdisciplinary Research in Basic Sciences, Jamia Millia Islamia, Jamia Nagar, New Delhi 110025, India \\ ${ }^{2}$ Department of Clinical Microbiology and Infectious Diseases, School of Pathology, University of the Witwatersrand, \\ Johannesburg 2193, South Africa \\ ${ }^{3}$ Molecular Sciences Institute, School of Chemistry, University of the Witwatersrand, WITS, 2050 Johannesburg, South Africa \\ ${ }^{4}$ Department of Pharmacognosy, College of Pharmacy, King Saud University, Riyadh 11451, Saudi Arabia
}

Correspondence should be addressed to Md. Imtaiyaz Hassan; mihassan@jmi.ac.in

Received 7 May 2020; Revised 14 June 2020; Accepted 24 June 2020; Published 17 July 2020

Guest Editor: Marcos R. de Oliveira

Copyright (c) 2020 Preeti Gupta et al. This is an open access article distributed under the Creative Commons Attribution License, which permits unrestricted use, distribution, and reproduction in any medium, provided the original work is properly cited.

\begin{abstract}
Calcium/calmodulin-dependent protein kinase IV (CaMKIV) is an upstream regulator of CaMKK-CaMKIV signaling cascade that activates various transcription factors, thereby regulating several cellular activities including, neuronal communication and immune response. Owing to the abnormal expression in cancer and neurodegenerative diseases, the CaMKIV has been considered a potential drug target. In the present study, we checked the binding affinity of plant-derived natural compounds viz., quercetin, ellagic acid (EA), simvastatin, capsaicin, ursolic acid, DL- $\alpha$-tocopherol acetate, and limonin towards CaMKIV. Molecular docking and fluorescence binding studies showed that EA and quercetin bind to the CaMKIV with a considerable affinity in comparison to other compounds. Enzyme inhibition assay revealed that both EA and quercetin inhibit CaMKIV activity with their $\mathrm{IC}_{50}$ values in the micromolar range. To get atomistic insights into the mode of interactions, inhibition mechanism, and the stability of the CaMKIV-ligand complex, a $100 \mathrm{~ns}$ MD simulation analysis was performed. Both EA and quercetin bind to the catalytically important residues of active site pocket of CaMKIV forming enough stabilizing interactions presumably inhibiting enzyme activity. Moreover, no significant structural change in the CaMKIV was observed upon binding of EA and quercetin. In conclusion, this study illustrates the application of phytoconstituents in the development of therapeutic molecules targeting CaMKIV having implications in cancer and neurodegenerative diseases after in vivo validation.
\end{abstract}

\section{Introduction}

Calcium/calmodulin-dependent kinase IV (CaMKIV), a member of the Ser/Thr protein kinase family, is an integral part of $\mathrm{Ca}^{2+}$-triggered CaMKK-CaMKIV signaling cascade where it regulates the phosphorylated-mediated activation of various transcription activators, such as JUN, CREB1, MEF2D, and RORA [1]. These downstream targets of CaMKIV further regulate various physiological processes including neuronal communication, inflammation, immune response, and memory consolidation [2]. It regulates cell motility, survival, cell cycle progression, and apoptosis via phosphorylation/dephosphorylation events $[3,4]$. CaMKIV shows high expression levels in the cerebellum, thymus, T-lymphocytes, and testis $[5,6]$. Under the basal conditions, the activation of CaMKIV is in the fine regulation of intracellular $\mathrm{Ca}^{2+}$ concentration. Increased intracellular free calcium stimulates a signaling cascade whereby a ubiquitously expressed protein calmodulin (CaM) binds to $\mathrm{Ca}^{2+}$, and $\mathrm{Ca}^{2+} / \mathrm{CaM}$ complex further interacts with CaMKIV and alters its functionality [7]. Intracellular $\mathrm{Ca}^{2+}$ levels have been identified as a crucial factor in the regulation of wide arrays of cell death modalities such as necrosis, necroptosis, and apoptosis $[8,9]$. It has been established that oxidative stress promotes the activation of CaMKIV which in turn induces the activation of antiapoptotic signaling cascades including ERK, AKT, and NF- $\kappa \mathrm{B}$ in various 
cell types. In conjunction with this, Rodriguez-Mora et al. has found that the inhibition of CaMKIV enhances the $\mathrm{H}_{2} \mathrm{O}_{2}$ induced apoptosis in breast cancer (MCF-7) cells [10].

Many reports have shown the abnormal expression of CaMKIV in various malignancies, including cancer of the lung, prostate, liver, and ovary [11-13]. In addition, the overexpression of CaMKIV is associated with systemic lupus erythematosus, cerebral hypoxia, ischemic stroke, and infectious diseases [14-16]. The involvement of CaMKIV and related kinases in the progression of cancers and neurological diseases makes it an attractive drug target $[1,17]$.

To date, chemotherapy is a common mode for cancer therapy. However, a concoction of drugs used in chemotherapy imposes serious threats upon long-term usage. Thus, it is imperative to devise alternative strategies that are effective in handling cancer and at the same time are clinically safe. For decades, plant-derived natural products have been investigated as promising therapeutic agents to combat cancer, neurodegenerative and cardiovascular diseases [18-20]. Natural compounds evoke diverse processes such as autophagic cell death, apoptosis, and programmed necrosis to elicit anticancerous activity $[19,21]$. The active ingredients of plant-derived formations are known to possess antioxidant, antidiabetic, anti-inflammatory, antibacterial, antiviral, and hepatoprotective effects [22-24]. Moreover, natural products overcome the disadvantages associated with conventional chemotherapy such as limited bioavailability and undesirable side effects [25]. An estimate by Alamgir [26] reveals that approximately 605 of the drugs that are available these days including paclitaxel, artemisinin, reserpine, and camptothecin were obtained from natural products either directly or indirectly.

Several studies exist where protein and phospholipid kinases have been targeted with the natural compounds. Most of the protein kinase inhibitors isolated from plants are polyphenols, mainly belonging to flavonoids [19-21, 27, 28]. However, there are representatives of nonflavonoids also that show inhibitory effects for human kinases, including resveratrol, caffeic acid, and gallic acid [29, 30]. Our previous studies demonstrated the binding and inhibition of CaMKIV by various natural compounds including hesperidin, $\beta$-carotene, and vanillin [31-33]. These compounds also showed the antiproliferative effect in breast (MCF-7), liver (HuH7 and HepG2), and neuroblastoma cancer cells (SH-SY5Y). These phytochemicals showed antiproliferative properties via inducing apoptotic pathways in cancerous cells.

Here, we studied a series of plant-derived natural compounds to assess their inhibitory effect towards CaMKIV [34]. The compounds showing best binding and inhibitory potential validated through experimental, docking, and molecular dynamics (MD) simulation studies. A detailed investigation of the binding mechanism provides atomistic insights into the mechanism of inhibition which may be further exploited for the design and development of clinical leads to address cancer and CaMKIV-associated diseases.

\section{Materials and Methods}

2.1. Materials. Isopropyl $\beta$-D-1-thiogalactopyranoside (IPTG), quercetin, ursolic acid, capsaicin, limonin, ellagic acid, simva- statin, DL- $\alpha$ tocopherol acetate, and dimethyl sulfoxide (DMSO) were procured from Sigma Aldrich (St. Louis, MO). Ni-NTA resin was bought from Thermo Scientific (USA). BIOMOL ${ }^{\circledR}$ Green reagent was bought from Enzo (New York, USA). All other reagents used were of analytical grade.

2.2. Molecular Docking Studies. The three-dimensional crystal structure of the CaMKIV enzyme was retrieved from the Protein Data Bank (https://www.rcsb.org/structure/2W4O). The structure of the enzyme was preprocessed, minimized, and refined using the Protein Preparation Wizard implemented in the Schrödinger suite [35-37]. This is associated with removing crystallographic waters, missing side chain or hydrogen atoms were added, and an accurate charge and protonation state was consigned to the enzyme structure consistent to $\mathrm{pH} 7.0$ considering the applicable ionization states for the acidic as well as basic amino acid residues. The structure was subsequently subjected to energy minimization using the OPLS-2005 force-field [38] with an RMSD cut-off value of $0.30 \AA$ to relieve the steric clashes among the residues due to the addition of hydrogen atoms.

The structure of quercetin (PubChem ID: 5280343) and EA (PubChem ID: 5281855) was retrieved from the PubChem database. These compounds were prepared using the LigPrep module implemented in the Schrödinger package [39]. This involved the addition of hydrogen atoms adjusting realistic bond lengths and angles, correct chiralities, ionization states, tautomers, stereo chemistries, and ring conformations. The OPLS-2005 force field was used to assign partial charges to the structure of the compounds. The compounds were subjected to energy minimization until average RMSD touched $0.001 \AA$ A. Epik [39] ionization tool was used to set the ionization state at the neutral $\mathrm{pH}$.

Molecular docking was performed using the Glide [40] docking tool of Maestro 11.6 to identify the best binding energies suitable for the CaMKIV enzyme. Receptor grid was generated as center coordinates $(X=7.37, Y=10.78$, and $Z=15.03$ ) using two cubical boxes having a common centroid to organize the calculations: a larger enclosing and a smaller binding box with dimensions of $15 \times 15 \times 15 \AA$. The grid box was centered on the centroid of the ligands in the complex, which was sufficiently large to explore a larger region of the enzyme structure. The ligands were docked by using the "Extra-Precision" mode (XP) protocol. The considered compounds were analyzed based on the docking scores and XP-G Scores.

2.3. MD Simulations. Molecular dynamics (MD) simulation was performed to see structural dynamics in the proteinligand complex. The AMBER 18 package [41] was used to execute MD simulations on all the prepared systems using the GPU-accelerated simulation engine PMEMD. The atomic partial charges for the ligands were assigned using the ANTECHAMBER module employed in general amber force field (GAFF) [42, 43]. To entirely solvate each system in a TIP3P virtual box filled with water molecules within $8 \AA$ box edge, the LEaP [44] component of AMBER 18 was utilized. The $\mathrm{Cl}^{-}$and $\mathrm{Na}^{+}$counter ions were added to approve the system neutralization before the production phase using 
the same LEaP component. A partial minimization of 1500 steps was achieved with $500 \mathrm{kcal} / \mathrm{mol}$ restraint potential gradient. The next full minimization of 1000 steps was further carried out by a conjugate gradient process by eliminating all the restraints applied before. Each system was then gradually being thermalized from $0 \mathrm{~K}$ to $300 \mathrm{~K}$ for $50 \mathrm{ps}$, assuring that each simulated system kept a stable volume and number of atoms. A potential harmonic restraint of $10 \mathrm{kcal} / \mathrm{mol}$ in combination with the collision frequency of 1 ps was applied to the solutes of all the systems. The equilibration of each system was performed by employing 500 ps equilibration step by ensuring $300 \mathrm{~K}$ constant temperature. NPT (isobaric-isothermal ensemble) was used to preserve a constant number of atoms and pressure within each system. The pressure was kept at 1 bar on each system using the Berendsen barostat. Ultimately, the production phase of $100 \mathrm{~ns}$ MD simulation was implemented on all the systems by integrating the SHAKE algorithm to restrict the hydrogen bonds [45].

2.4. Postdynamic Analyses. The coordinates of enzymes bound with the inhibitors were further saved after every $1 \mathrm{ps}$, and the trajectory curves were calculated using the CPPTRAJ [46] module integrated into the AMBER 18 package. The RMSD of $\mathrm{C}^{\alpha}$ atoms, RMSF of each residue in the complex $R_{\mathrm{g}}$, SASA, intramolecular and intermolecular hydrogen bond formation, and thermodynamic calculations of all systems were calculated. We used Origin software and Gnuplot for MD trajectory analysis [47].

2.5. Binding Free Energy Calculations. The comparative binding free energies were analyzed using the Molecular Mechanics/Generalized Born Surface Area (MM/GBSA) binding free energy technique [48]. All solvent and counter ions were eliminated using the CPPTRAJ module. The binding free energies $\left(\Delta G_{\text {bind }}\right)$ were estimated with the MM-GBSA method for each system as below:

$$
\Delta G_{\text {bind }}=G_{\text {complex }}-G_{\text {protein }}-G_{\text {ligand }} \text {. }
$$

The free energy term, $\Delta G_{\text {bind }}$ is computed using the following equations:

$$
\Delta G_{\text {bind }}=\Delta E_{\text {gas }}+\Delta G_{\text {solvation }}-T \Delta S
$$

where

$$
\begin{gathered}
\Delta E_{\text {gas }}=E_{\text {int }}+E_{\mathrm{vdW}}+E_{\text {elec }}, \\
E_{\text {int }}=E_{\text {bond }}+E_{\text {angle }}+E_{\text {torsion }}, \\
G_{\text {solvation, } G B}=G_{\mathrm{GB}}+G_{\text {nonpolar,solvation }}, \\
G_{\text {nonpolar }}=\gamma S A S A+\beta .
\end{gathered}
$$

The gas-phase energy $\left(\Delta E_{\text {gas }}\right)$ is the total of the internal $\left(E_{\text {int }}\right)$, van der Waals $\left(E_{\mathrm{vdW}}\right)$, and Coulombic $\left(\Delta E_{\text {elec }}\right)$ energies, (Equation (3)). The solvation free energy is the combination of polar $\left(G_{\mathrm{GB}}\right)$ and nonpolar $\left(\Delta G_{\text {nonpolar }}, \Delta G_{\text {solvation }}\right)$ contributions (Equation (5)). The polar solvation $G_{\mathrm{GB}}$ contribution was calculated using the Generalized Born (GB) solvation model with the dielectric constant 1 for solute and 80.0 for the solvent. However, the nonpolar free energy contribution was assessed using (Equation (6)), where the surface tension proportionality constant, $\gamma$, and the free energy of nonpolar solvation of a point solute, $\beta$, were set to $0.00542 \mathrm{kcal} \mathrm{mol}^{-1} \AA^{-2}$ and $0 \mathrm{kcal} \mathrm{mol}^{-1}$, respectively. The SASA is calculated by the linear combination of the pairwise overlap (LCPO) model.

2.6. Dynamic of the Cross-Correlation Matrix. The dynamic cross-correlation matrix (DCCM) analysis was calculated to explore the variations and displacements in the $\mathrm{C}^{\alpha}$ atoms. Components for $i$ and $j$ cross-correlation $\mathrm{C}^{\alpha}$ atoms are presented underneath:

$$
c_{i j}=\frac{<\Delta r_{i} \cdot \Delta r_{j}>}{\left(<\Delta r_{i}^{2}><\Delta r_{j}^{2}>\right)^{1 / 2}},
$$

where $\Delta r_{i, j}$ is the motion of $i^{\text {th }}$ and $j^{\text {th }}$ atom mean point, and angle braces indicate the interval throughout the complete curves. All correlated actions are denoted by $C_{i j}=1$ whereas $C_{i j}=-1$ indicated highly anticorrelated motions over the course. The divergence of motion from 1 and -1 designate that $i$ and $j$ motions are correlated and anticorrelated correspondingly. The DCCM was analyzed using the CPPTRAJ module implemented in Amber 18 and all the matrices schemed in Origin software.

2.7. Expression and Purification of CaMKIV. The plasmid containing CaMKIV kinase domain cDNA (pET28a (+)) was transformed into an expression vector, BL21 (DE3) strain of Escherichia coli. The transformed E. coli cells were grown in Luria broth containing $50 \mu \mathrm{g} / \mathrm{ml}$ kanamycin till the $\mathrm{A}_{600}$ reaches $\sim 0.6-0.8$. Subsequently, $0.25 \mathrm{mM}$ IPTG was added to the culture broth followed by an incubation of $3-4 \mathrm{hrs}$ at $37^{\circ} \mathrm{C}$ for the expression of recombinant protein. The detailed protocol for the purification of CAMKIV is described in our previous communications [18, 49]. Briefly, the cells were lysed by sonication, and inclusion bodies were prepared and subsequently solubilized using $1.0 \%$ of $\mathrm{N}$ laurousyl sarcosine in CAPS buffer at $\mathrm{pH} 11.0$. The protein was purified in a single step utilizing Ni-NTA affinity chromatography. The protein was eluted using $250 \mathrm{mM}$ imidazole and dialyzed thoroughly against dialyzing buffer $(20 \mathrm{mM}$ phosphate buffer, $\mathrm{pH} 7.4,100 \mathrm{mM} \mathrm{NaCl}$ ) for 48 hours with 5-6 successive buffer changes yielding the refolded protein. A molar absorption coefficient of $47245 \mathrm{M}^{-1} \mathrm{~cm}^{-1}$ at $280 \mathrm{~nm}$ was used to calculate the protein concentration using Jasco V-660 UV-visible spectrophotometer (Japan).

2.8. Fluorescence Spectroscopy. Fluorescence binding measurements were carried out on the Jasco fluorescence spectrophotometer (Jasco, Japan) equipped with a thermostated Peltier device that controls the temperature at $25 \pm 0.1^{\circ} \mathrm{C}$. A $5 \mathrm{~mm}$ quartz cuvette was used to perform all measurements. The compounds were dissolved in DMSO to prepare a $1 \mathrm{mM}$ stock solution. CaMKIV $(4.0 \mu \mathrm{M}$ in $20 \mathrm{mM}$ phosphate buffer, 
$\mathrm{pH}$ 7.4) in the cuvette was titrated with increasing concentrations of the ligand from the stock solution. After each addition of ligand, the solution was properly mixed and the fluorescence emission spectrum was acquired from 300 to $400 \mathrm{~nm}$ wavelength range after exciting the protein at $280 \mathrm{~nm}$. Both excitation and emission slits were set at $5 \mathrm{~nm}$ each. The corresponding blank was subtracted from each spectrum to obtain the final emission spectra. Subsequently, the Stern-Volmer equation was employed to analyze the data for the estimation of the number of binding sites $(n)$ per molecule of CaMKIV and binding constant $\left(K_{a}\right)[50,51]$.

2.9. Kinase Inhibition Assay. The ATPase activity of CaMKIV with ligands was evaluated by malachite green reagent (BIOMOL ${ }^{\circledR}$ Green reagent) -based microtitre plate assay. CaMKIV $(2.0 \mu \mathrm{M})$ was mixed with increasing concentrations of quercetin and EA and incubated for $30 \mathrm{~min}$ at $25^{\circ} \mathrm{C}$. Subsequently, a freshly prepared ATP solution $(200 \mu \mathrm{M})$ was added followed by incubation for $30 \mathrm{~min}$ at $25^{\circ} \mathrm{C}$. BIOMOL ${ }^{\circledR}$ Green reagent was then added gently to terminate the reaction followed by further incubation of $15 \mathrm{~min}$ for green color development. The microtitre plate was then read on ELISA reader at $620 \mathrm{~nm}$. The standard phosphate curve was prepared as per the manufacturer's protocol to calculate the kinase activity in terms of inorganic phosphate released from ATP upon catalysis. Measurements in triplicates were performed as described [52].

\section{Results and Discussion}

3.1. Molecular Docking Analysis. The natural compounds such as quercetin, EA, simvastatin, capsaicin, ursolic acid, DL- $\alpha$-tocopherol acetate, and limonin were retrieved from the PubChem database to probe their potential interactions with the binding pocket of CaMKIV by molecular docking. We got an optimized orientation of ligands in the active site cavity of protein by minimizing the overall energies of the respective system. The calculated values of binding energies of all the compounds are shown in Table S1. Quercetin and EA showed the best binding energy and thus considered as promising inhibitors of CaMKIV (Figure 1). The quercetinCaMKIV and EA-CaMKIV docked complexes showed significant binding energy values, -9.78 and $-9.31 \mathrm{kcal} / \mathrm{mol}$, respectively (Table S1.

The interaction analysis of docked complexes suggests that both quercetin and EA form various stabilizing interactions including hydrophobic and $\mathrm{H}$-bonded interactions with the residues of the active pocket of CaMKIV (Figure 2). Quercetin formed three hydrogen bonds with the residues of the active site pocket of CaMKIV. The catechol ring of quercetin forms two hydrogen bonds with polar side chains of Glu76, while the third hydrogen bond was formed by the hydroxyl $(-\mathrm{OH})$ group of another ring with Asp142 (Figure 2(a)). In addition, Glu125 is creating $\pi$-sigma interaction with the aromatic ring; whereas, a set of hydrophobic residues including, Val27, Ala40, Leu128, and Ala141 are forming a weak network of $\pi$-alkyl interactions with the catechol ring of quercetin.

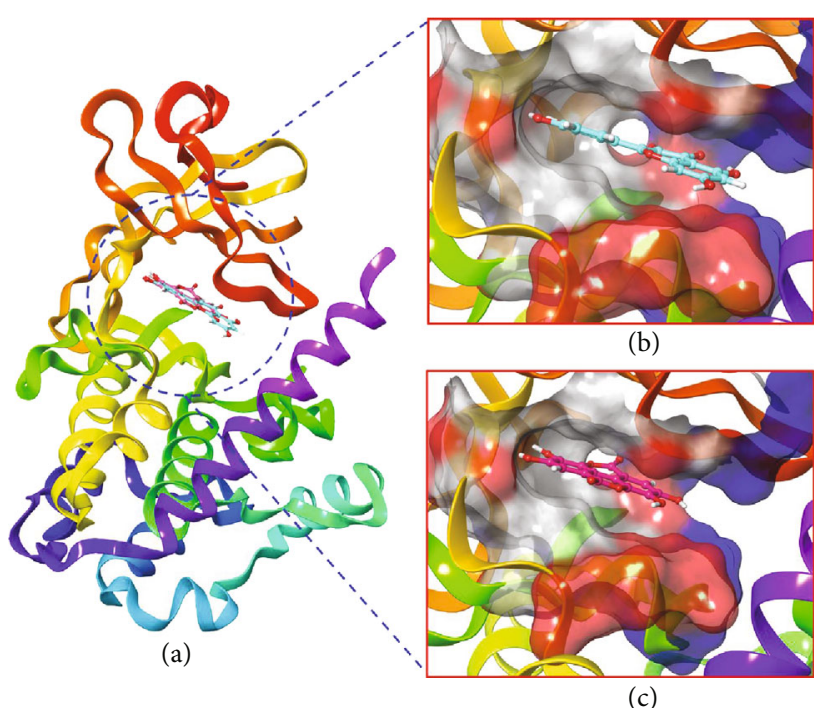

FIgURE 1: (a) Structural representation of CaMKIV in complex with quercetin (cyan color) and EA (pink color). (b) Close up surface view of the deep binding pocket of CaMKIV-accommodated quercetin. (c) Close up surface view of the deep active pocket accommodated with the EA (colored by residue type).

The structure of EA is composed of a network of four aromatic rings that serve as a hydrophobic moiety, whereas the side chain lactone and hydroxyl groups act as an electron acceptor and H-bonding sites, respectively [53]. The polar sidechain of Glu76 forms two hydrogen bonds, while the backbone amide group of Val78 forms one hydrogen bond with the hydroxyl groups of EA (Figure 2(b)). A subset of hydrophobic residues having Val27, Ala40, Leu128, and Ala141 are forming a compact network of $\pi$-alkyl noncovalent interactions with the bulky aromatic rings of EA. Additionally, Asp142 is involved in stabilizing the complex via van der Waals interactions with EA.

Overall, the structural and interaction analyses revealed that both quercetin and EA bind strongly to the deep active site pocket of CaMKIV through hydrogen-bonded, $\pi$-sigma, and $\pi$-alkyl interactions that might be responsible for the modulation of enzyme activity.

3.2. Comparative Structural Dynamics. The conformational changes in the enzyme structure are precisely coupled with their biological functions [54]. Any alteration or interruption in enzymes' structural integrity could have a considerable impact on its function [55]. The binding of small molecule inhibitors influences the mode of action of enzymes that are implicated in disease pathways; hence, it is required to evaluate the structural dynamics and structural changes associated with the inhibitory activity of these compounds [56]. The calculation of a time variable concerning the root means square deviation (RMSD) across $\mathrm{C}^{\alpha}$ atoms from generated trajectories were executed to determine the consistency and efficiency of the simulated CaMKIV in complex with quercetin and EA along with the CaMKIV alone [57]. The alterations in the RMSD values were shown in the plot throughout the simulation period, possible conformational 


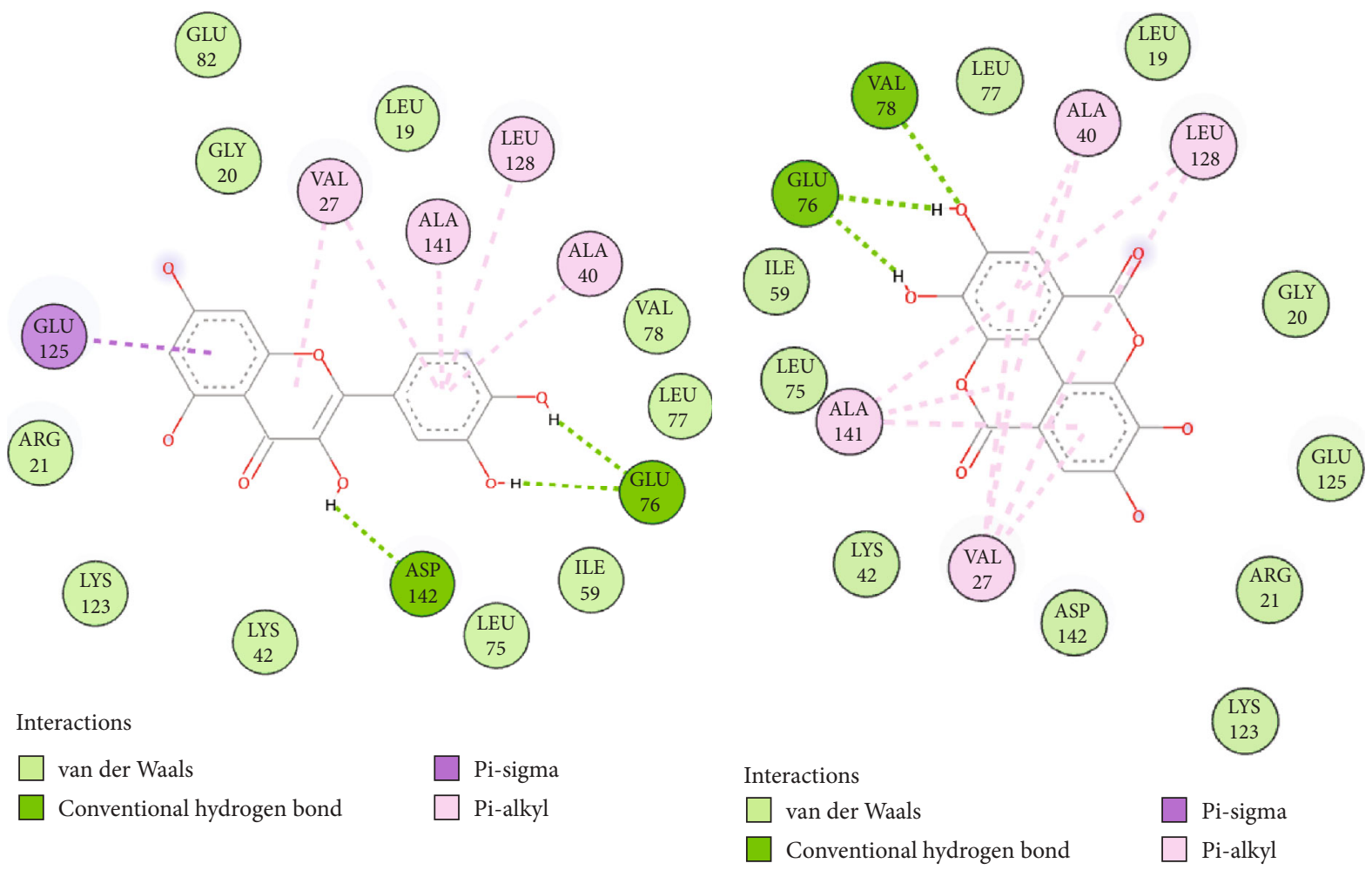

(a)

(b)

FIGURE 2: Docked poses of CaMKIV with the selected inhibitors. Molecular interaction of (a) quercetin and (b) EA with the active pocket of CaMKIV enzyme.

changes within the structure of an enzyme upon ligand binding. As Figure 3(a) suggests, all the systems were stabilized and achieved a convergence after $40 \mathrm{~ns}$ of the production phase of the simulation. Quercetin-CMKIV complex exhibited the lowest average RMSD of $2.47 \AA$, whereas EACaMKIV and apo CaMKIV showed an average RMSD of $2.49 \AA$ and $2.51 \AA$, respectively. The RMSD plots indicate that both quercetin and EA exhibit almost the lowest change in the $\mathrm{C}^{\alpha}$ backbone atoms, suggesting binding of these compounds induces stability to the CaMKIV structure. The overall inhibitory activity of CaMKIV by quercetin and EA was supported by structural stabilization and subsequent inhibition in the enzyme activity. The stability of quercetin and EA were further assessed throughout the simulation time as their related activities may correspond to the active pocket residues.

The flexible regions of a protein provide flexibility which is sometimes very important for the biological activity especially in the case of the enzyme [58]. Hence, ligand-enzyme binding may be observed concerning the alteration in flexibility expressed in terms of root mean square fluctuation (RMSF). To ascertain the flexibility and rigidity of all the residues in CaMKIV upon quercetin and EA binding, RMSF values for $\mathrm{C}^{\alpha}$ atoms were estimated through the MD simulations produced trajectories over $100 \mathrm{~ns}$ of the period. As illustrated in Figure 3(b), the quercetin-CaMKIV complex revealed a minimum fluctuation in the residues with an average value of $8.45 \AA$. Standard RMSF values of $11.14 \AA$ and $12.29 \AA$ were observed in EA-CaMKIV complex and apo CaMKIV, respectively. These observations revealed that quercetin exhibited the lowest fluctuation which could be indicative of a better binding in comparison to the EA. This considerable decrease may be associated with structural deactivation that was assured as a result of the prominent binding of compounds in the active pocket of CaMKIV. A reduced fluctuation of all the residues might have favored CaMKIV inhibition.

To further complement our analysis, we have estimated $R_{\mathrm{g}}$ value, related to the intact conformational changes in the enzyme structure upon ligand binding. It also uncovers the compactness, stability, and folding behavior of enzyme structure [59]. We assessed the compactness of quercetin, EA, and apo CaMKIV complexes by evaluating their $R_{\mathrm{g}}$ values. The average $R_{\mathrm{g}}$ values for quercetin-CaMKIV, EACaMKIVcomplexes, and apo CaMKIV were calculated as $37.12 \AA, 41.39 \AA$, and $36.12 \AA$, respectively. Figure $3(\mathrm{C})$ showed a slight alteration in the compactness in the presence of two compounds. Quercetin showed a higher fluctuation before $20 \mathrm{~ns}$, but afterward, it was stable. EA also exhibited similar behavior as it showed stability until $60 \mathrm{~ns}$ and after that higher fluctuation was noted. The lowest $R_{\mathrm{g}}$ value was exhibited by the apo CaMKIV enzyme, suggesting no significant change upon ligand binding to the CaMKIV enzyme.

We further analyzed changes in the SASA values to define the hydrophobic and hydrophilic function of residues and forces exposed to the solvent in the simulation period [60, 61]. The rapid and constant values of SASA is advantageous 


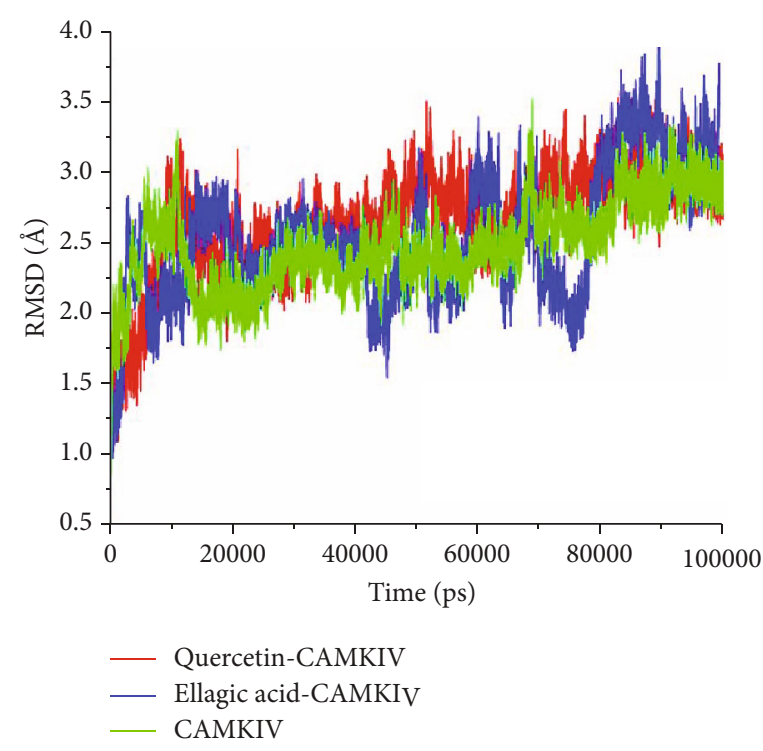

(a)

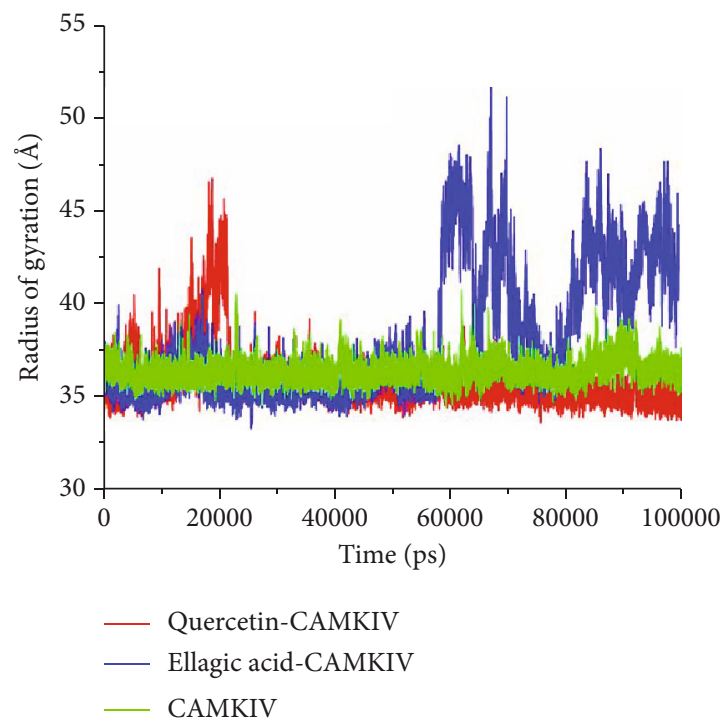

(c)

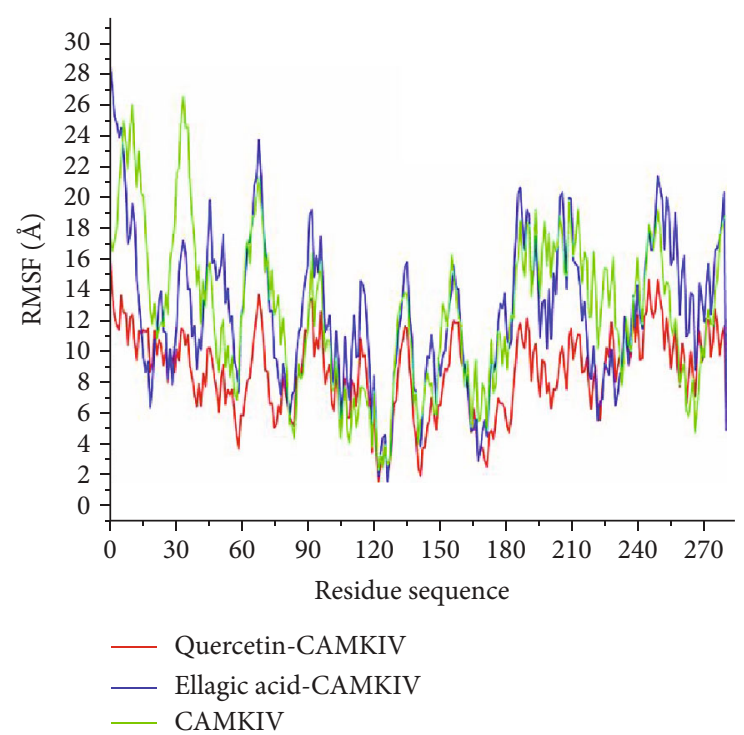

(b)

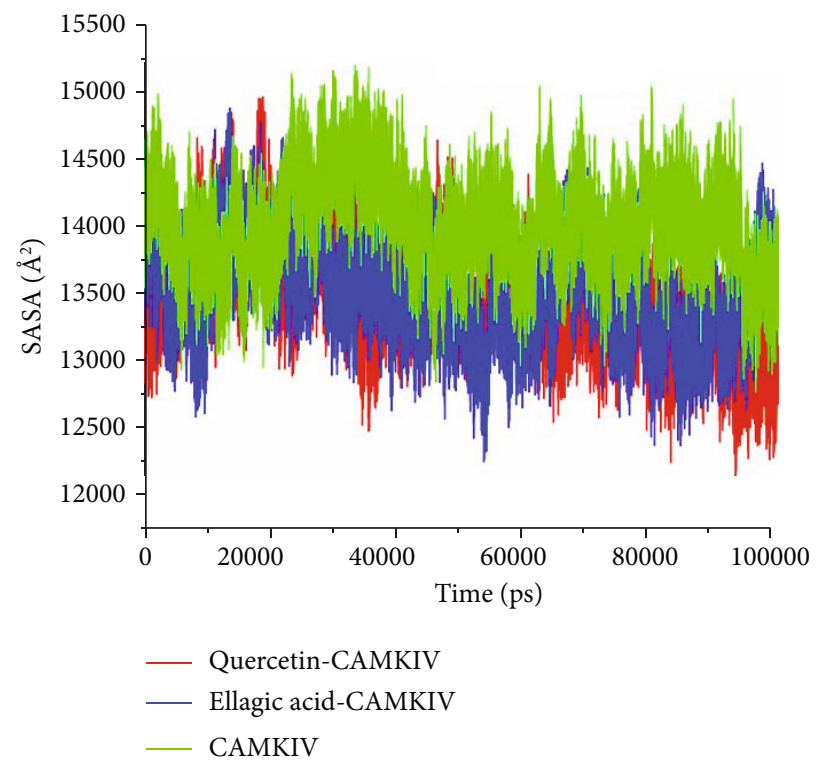

(d)

Figure 3: Structural dynamics of CaMKIV enzyme-ligand complexes. (a) RMSD, (b) RMSF, (c) $R_{\mathrm{g}}$ values, and (d) SASA values across C ${ }^{\alpha}$ backbone in $\AA$ of quercetin- CaMKIV, EA-CaMKIV, and apo CaMKIV; in $\AA$ across $\mathrm{C}^{\alpha}$ backbone of all the three conditions calculated during the $100 \mathrm{~ns}$ of MD trajectories.

in the energetic assessment of biomolecules. The connection among native hydrophobic contacts inside the enzyme structure is an important intermolecular interaction which is associated with the enzyme inhibition. Hydrophobic interaction formed between the nonpolar residues endorses constancy of the enzyme structure within the solution by preserving the nonpolar residues inside the hydrophobic core away from an aqueous solution [62]. Figure 3(D) illustrates overall changes in the SASA values for all the complexes analyzed during $100 \mathrm{~ns}$ of $\mathrm{MD}$ simulation time. The average SASA value for the quercetin-CaMKIV complex was $13512 \AA^{2}$ exposed to the solvent system. The total value of SASA of $13683 \AA^{2}$ and $14079 \AA^{2}$ was calculated for EA-CaMKIV complex and apo CaMKIV, respectively. Such a difference in the values of SASA is related to the extent of binding and their subsequent impact on the enzyme inhibition. The assessment of SASA in the quercetin-bound complex revealed a better exposure to the solvent and consequently favored the increased inhibitory potential of quercetin over other complexes.

3.3. Analysis of Intermolecular and Intramolecular Hydrogen Bonds. We have calculated the intramolecular and intermolecular hydrogen bonding for CaMKIV alone and in complex with quercetin and EA to confirm the overall stability and strength of interactions [40]. Figure 4 provides a detailed 


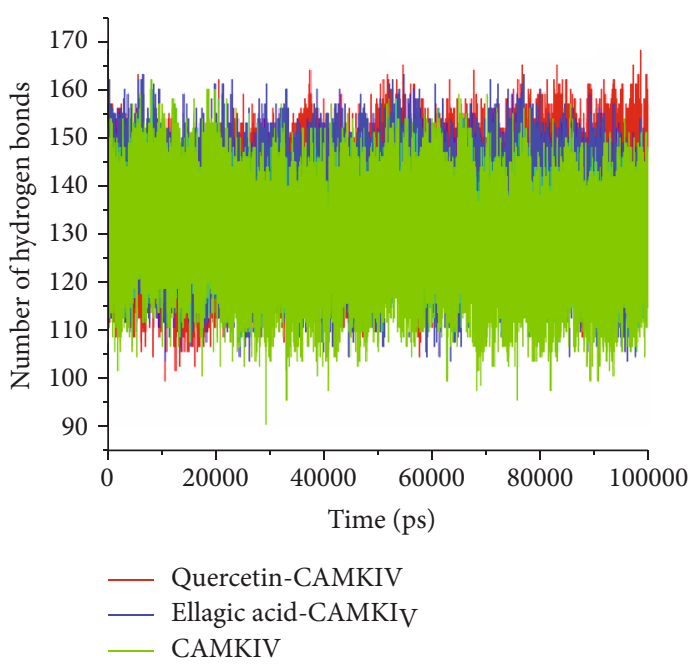

(a)

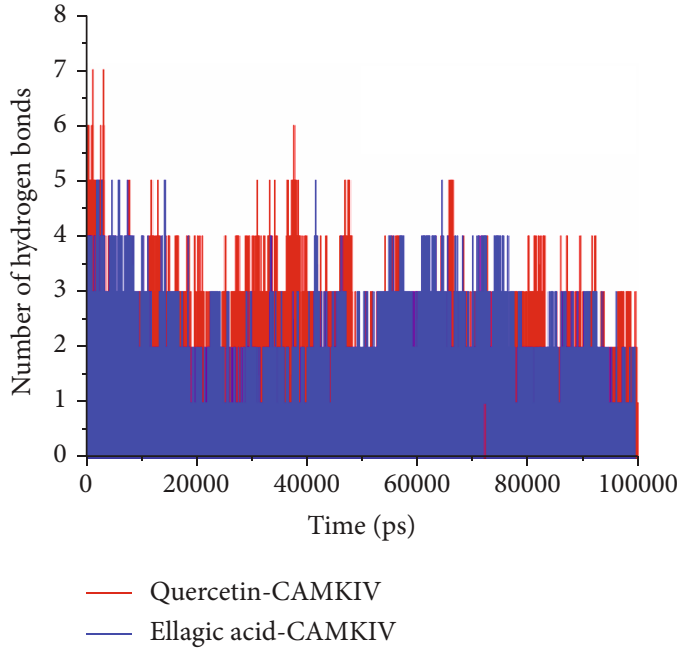

(b)

FIgURE 4: Hydrogen bond analysis. (a) Intramolecular hydrogen bonds in quercetin, EA-CaMKIV complexes, and apo CaMKIV enzyme. (b) Intermolecular hydrogen bonds in quercetin, and EA-bound CaMKIV complexes calculated after 100 ns MD simulation.

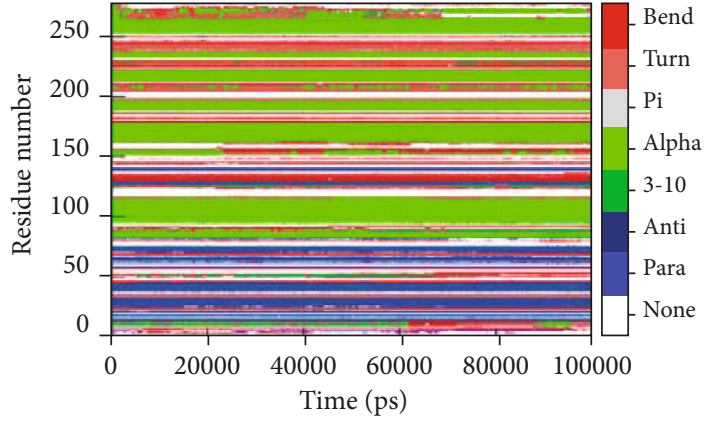

(a)

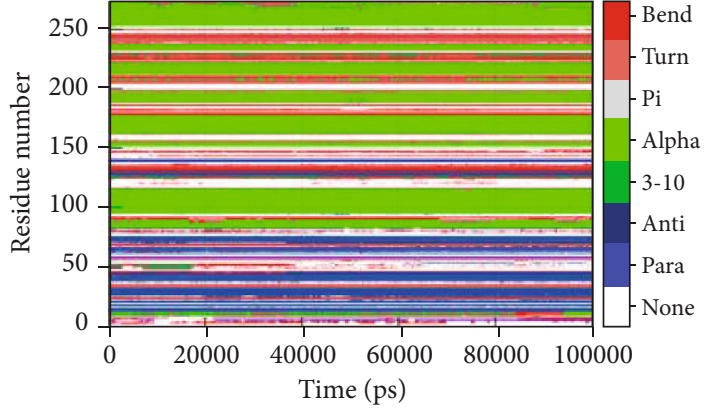

(b)

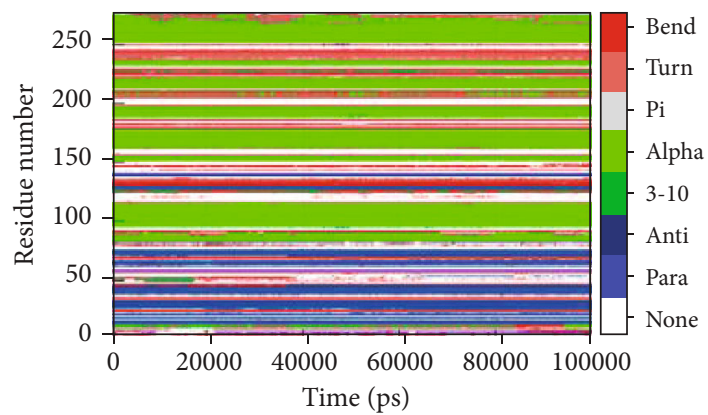

(c)

FIgure 5: Secondary structure analysis. (a) Apo CaMKIV enzyme. (b) Quercetin-bound CaMKIV enzyme. (c) EA-bound CaMKI enzyme.

information of the hydrogen bonding pattern of proteinligand interactions. In the quercetin-CaMKIV complex, an average number of intramolecular hydrogen bond formation was noted as 137; however, in the case of EA-CaMKIV and apo CaMKIV, it was 135 and 130, respectively, during the $100 \mathrm{~ns}$ simulation time. On the other hand, the intermolecular hydrogen bond formation in the active pocket of CaMKIV enzyme observed to be 3-4 bonds with higher fluctuations and 2-3 bonds with the least fluctuations in quercetin and EA complexes.
3.4. Secondary Structure Analysis. To analyze the alterations incurred due to ligand binding to the CaMKIV enzyme, the dynamics of secondary structure contents were measured. The structural elements like $\alpha$-helix, $\beta$-sheet, and turn in CaMKIV were classified into individual amino acid residues for every time step; thus, an average of amino acid residues involved in the formation of secondary structure was calculated. Figure 5(a) shows that in apo CaMKIV marginally a higher fluctuation in the turn in comparison to the other structural components. A slight increase was observed in 


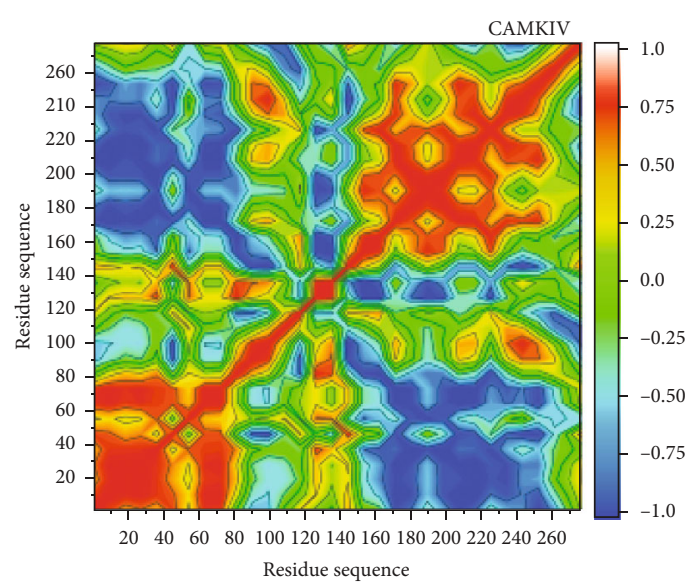

(a)

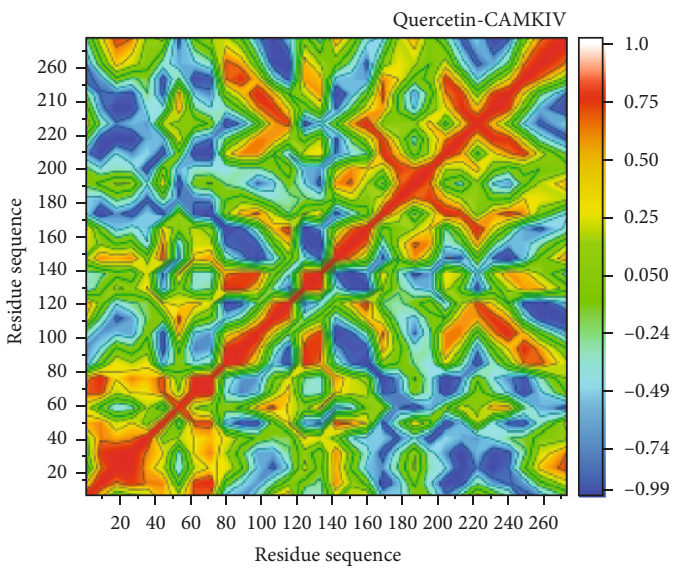

(b)

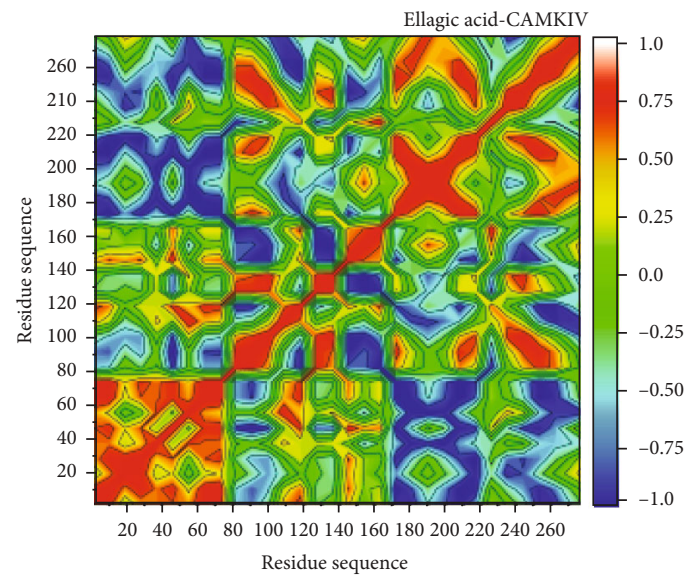

(c)

Figure 6: Dynamic cross-correlation matrix analyses. (a) Apo CaMKIV enzyme. (b) Quercetin-bound CaMKIV complex. (c) EA-bound CaMKIV enzyme inhibition. Numbers closer to 1 indicate high correlation, while those closer to -1 indicate anticorrelation between pairs of residues.

the $\alpha$-helix and bend in quercetin and EA-bound CaMKIV complexes (Figures 5(b) and 5(c)). These higher components are suggestive of a stabilized binding of the ligands to the enzyme. Although secondary structure components in CaMKIV do not contribute to any major changes showing enhanced flexibility, compactness, and stability of both the compounds.

3.5. Dynamic Cross-Correlation Matrix Analysis. Distinct enzyme dynamics among apo CaMKIV, quercetin-CaMKIV, and EA-CaMKIV complexes were measure by plotting dynamic cross-correlation matrix for the correlated and anticorrelated motions of all residues. CaMKIV was separated into different groups with positive and negative movements of amino acid residues. The apo CaMKIV was showing both positive and negative correlation between the residual motions (Figure 6(a)). As shown in Figures 6(b) and $6(\mathrm{c})$, significant differences were observed between quercetin and EA-CaMKIV complexes. There was a less positive correlation in the quercetin-bound complex as compared to the EA complex. The apo CaMKIV and EACaMKIV complex appeared to be similar, indicative that inhibition through EA induced noteworthy changes in
CaMKIV dynamics. Few residues (1-40 and 260-302) in quercetin-CaMKIV complex showed a highly positive correlation of movements; therefore, they might be contributing to the overall dynamics of the CaMKIV enzyme.

3.6. Thermodynamics Free Energy Landscape. The thermodynamic energy contribution of a ligand to the overall binding free energy of the complex is directly related to the structural stability of the ligand in the active pocket of the enzyme. The molecular interactions of residues in the active pocket engage significantly in the stability, binding affinity, and selectivity of the ligand. Thus, it was essential to assess the binding affinity of quercetin and EA towards the CaMKIV enzyme using the MM/GBSA approach to determine the effect of these compounds. The obtained results are displayed in Table 1.

The free binding energy $\left(\Delta G_{\text {bind }}\right)$ of quercetin-bound CaMKIV complex was noted to be the highest energy with an average value of $-29.15 \mathrm{kcal} / \mathrm{mol}$ relative to the EACaMKIV complex with $-23.75 \mathrm{kcal} / \mathrm{mol}$. These total binding energies of the complexes were indicative of the effective binding of quercetin to its target enzyme. We further assessed other constituents of the free binding energy coupled with enzyme-inhibitor binding (Table 1). It was observed that 
TABLE 1: MM/GBSA-based binding energy profile of CaMKIV in complex with quercetin and EA.

\begin{tabular}{lccccccc}
\hline Complex & $\Delta E_{\mathrm{vdW}}$ & $\Delta E_{\text {elec }}$ & $\Delta G_{\text {gas }}$ & $\Delta G_{\text {polar }}$ & $\Delta G_{\text {nonpolar }}$ & $\Delta G_{\text {solvation }}$ & $\Delta G_{\text {bind }}$ \\
\hline Quercetin-CaMKIV & -33.69 & -26.51 & -60.20 & 41.03 & -4.58 & 36.45 & -23.75 \\
EA-CaMKIV & -25.90 & -29.42 & -55.32 & 30.43 & -4.26 & 26.18 & -29.15 \\
\hline
\end{tabular}

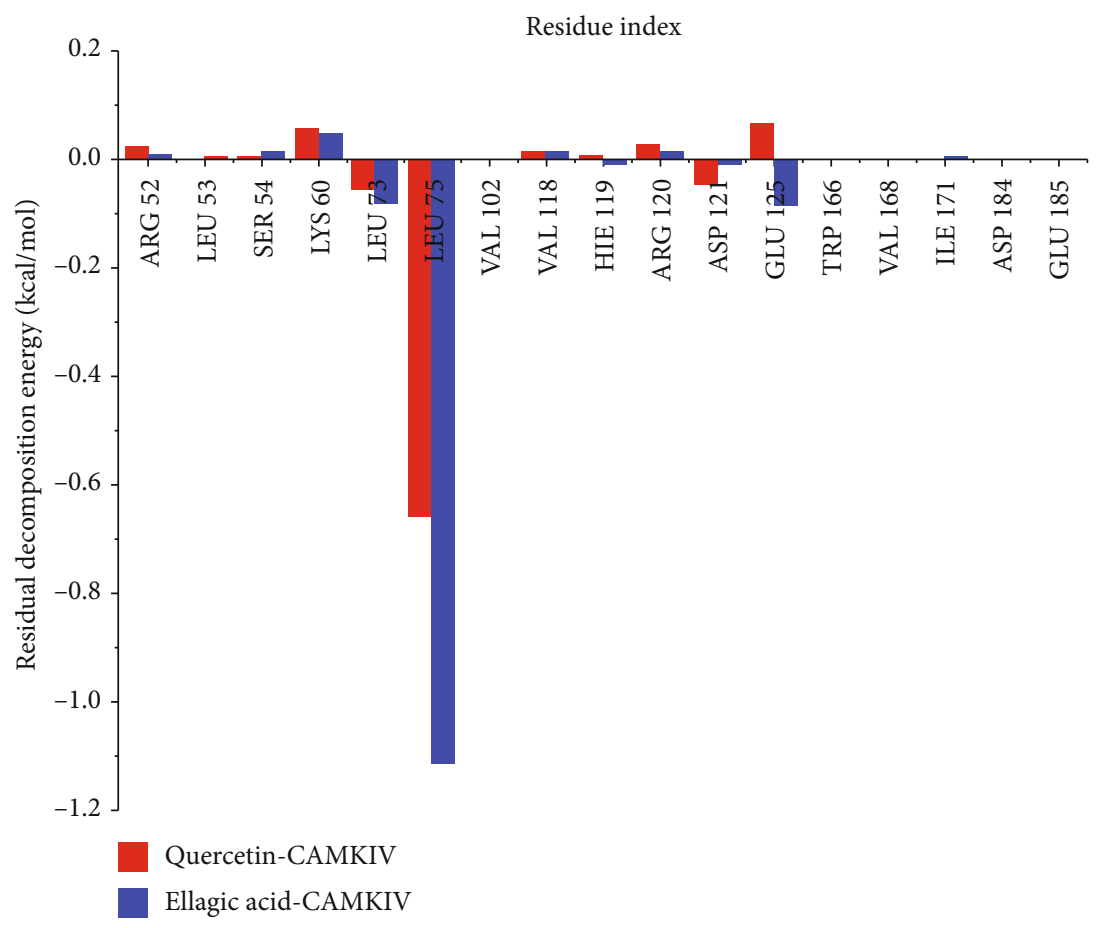

FIGURE 7: The per residue energy decomposition analysis graphs of quercetin and ellagic acid CaMKIV complexes, plotted after $100 \mathrm{~ns}$ MD simulations.

intermolecular van der Waals and gas-phase energies were more favorable in the EA-CaMKIV complex with the average values of -33.69 and $-60.20 \mathrm{kcal} / \mathrm{mol}$, whereas, these energies are slightly less in quercetin bound CaM$\operatorname{KIV}(-25.90$ and $-55.32 \mathrm{kcal} / \mathrm{mol})$. There was a significant difference of $-5.4 \mathrm{kcal} / \mathrm{mol}$ between the $\Delta G_{\text {bind }}$ energies of quercetin and EA complexes. The $\Delta G_{\text {solvation }}$ contributed to unfavorable binding of quercetin as it was the least energy with a value of $26.18 \mathrm{kcal} / \mathrm{mol}$ between the two complexes. However, the systematic motions of quercetin and EA from the solvent phase to the active pocket of CaMKIV-stimulated van der Waals and electrostatic interactions with the active site residues, but these interactions were not adequate to support completely in the binding of the CaMKIV enzyme as $\Delta G_{\text {bind }}$ contributes to the improved binding of these compounds. This analysis suggests that both quercetin and EA possess a significant binding affinity to the CaMKIV.

3.7. Per Residue Energy Decomposition Analysis. The binding free energy decomposition gives a deeper insight into the annotation of enzyme-ligand complexes generated through the trajectories by MD simulations. Herein, we have disintegrated the overall binding energies of complexes into per residual contribution by each amino acid residue present in the active pocket of the CaMKIV enzyme to get detailed insights into key residues involved in ligand binding. The interactions among active site pocket electronegative and electropositive residues improve ligand binding and its stabilization at the target site. This forms an improved intermolecular binding that upsurges the binding affinity of the ligand in the active pocket. Leu76 contributed with the lowest $\Delta G_{\text {bind }}$ with $-1.76 \mathrm{kcal} / \mathrm{mol}$ in EA binding, whereas this residue has contributed with slightly less $\Delta G_{\text {bind }}$ of $-0.67 \mathrm{kcal} / \mathrm{mol}$ in quercetin-bound complex (Figure 7). The $\Delta G_{\text {bind }}$ of another contributing residue Leu73 was also lower in the case of EA-CaMKIV with $-0.45 \mathrm{kcal} / \mathrm{mol}$; however, it is marginally less in the quercetin-CaMKIV complex $(-0.35 \mathrm{kcal} / \mathrm{mol})$. Arg52, Lys60, Val118, and Arg120 contributed to the binding of quercetin and EA with positive binding energies. Glu125 amino acid residue contributed to the negative energy of $-0.05 \mathrm{kcal} / \mathrm{mol}$ in $\mathrm{EA}$, whereas this residue was showing positive energy of $0.03 \mathrm{kcal} / \mathrm{mol}$ in quercetin-bound CaMKIV complex. Our analysis reveals residues contributing to the overall binding energies of both the complexes.

3.8. Fluorescence Binding Studies. Fluorescence quenching has long been utilized to measure the binding affinity of the protein with the ligand $[63,64]$. An array of molecular 


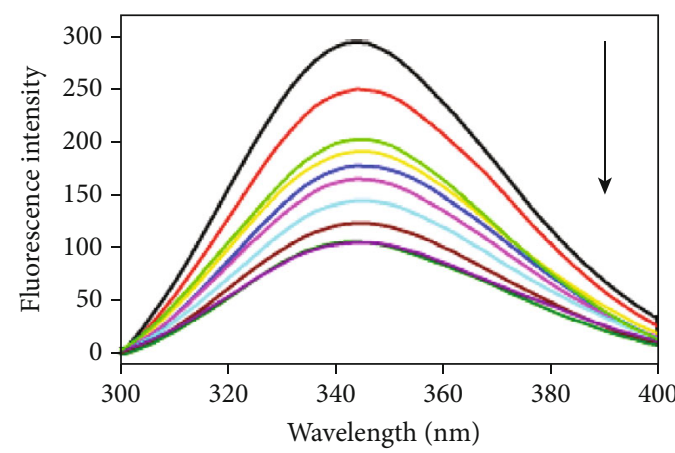

(a)

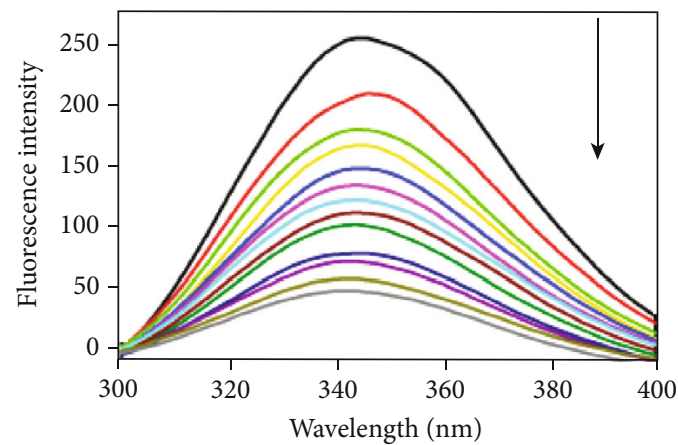

(c)

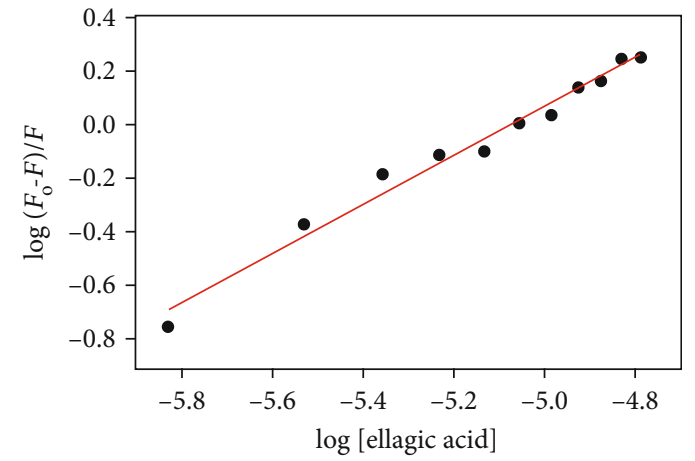

(b)

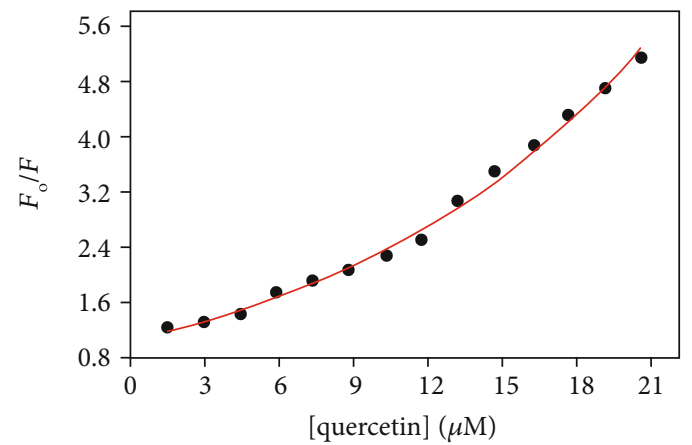

(d)

FIGURE 8: Fluorescence binding study of CaMKIV with quercetin and EA. (a) Fluorescence spectra of CaMKIV (4 $\mu$ M) with increasing concentrations of EA (from top to bottom) at pH 7.4. (b) Stern-Volmer plot obtained by plotting fluorescence intensity at $\lambda_{\text {max }}$ as a function of EA and fitting to Equation (1). (c) Fluorescence spectra of CaMKIV $(4 \mu \mathrm{M})$ with increasing concentrations of EA (from top to bottom) at pH 7.4. The excitation was kept constant at $280 \mathrm{~nm}$ and the emission spectra was acquired from 300 to $400 \mathrm{~nm}$. (d) Stern-Volmer plot obtained by plotting fluorescence intensity at $\lambda_{\max }$ as a function of quercetin fitting to Equation (2).

interactions such as intersystem crossing to the triplet state, formation of an excited charge-transfer complex, complex formation between the quencher and fluorophore at ground state, and molecular rearrangements can be attributed to fluorescence quenching [65].

To complement the in silico findings, we carried out fluorescence measurements to determine the binding affinity of CaMKIV with the selected natural compounds (Figure S1). CaMKIV $(4.0 \mu \mathrm{M})$ was titrated with an increasing concentrations of natural compounds from $1.0 \mathrm{mM}$ stock solution. Thereafter, fluorescence emission spectra were collected in the wavelength range of 300 to $400 \mathrm{~nm}$ after exciting the protein at $280 \mathrm{~nm}$. The titration and data acquisition was followed until the saturation point was achieved. Figure 8 and Figure S1 shows the emission spectra of CaMKIV in the presence of an increasing concentration of different ligands. The compounds showing good binding interaction with CaMKIV are quercetin and EA; a continual decline in fluorescence intensity was noticed with each successive addition of ligand (Figures 8(a) and 8(c)). In contrast, the other compounds showed less or no significant quenching effect indicating the absence of any strong binding interaction with the protein (Figure S1). For EA and quercetin, the fluorescence intensity at $\lambda_{\max }\left(F_{0} / F\right)$ was plotted against a ligand concentration and fitted to the Stern-Volmer equation to estimate binding constants (Ksv) (Figures 8(b) and 8(d)).
TABLE 2: Binding constants and inhibitory concentrations of EA and quercetin to CAMKIV.

\begin{tabular}{lcc}
\hline Compound & ${ }^{*}$ Binding constant $\left(K_{\mathrm{sv}}, \mathrm{M}^{-1}\right)$ & ${ }^{¥} \mathrm{IC}_{50}(\mu \mathrm{M})$ \\
\hline EA & $4.28 \pm 0.28 \times 10^{4}$ & $39.7 \pm 8.6$ \\
Quercetin & $7.82 \pm 0.54 \times 10^{2}$ & $61.3 \pm 5.4$
\end{tabular}

* Binding constant derived from fluorescence binding measurements. ${ }^{{ }^{\sharp}} \mathrm{IC}_{50}$ value determined from enzyme inhibition assay.

The following equations were used to analyze data depending on the resulting curves and quenching mechanism [66]:

$$
\begin{gathered}
\log \left(F_{0}-F / F\right)=\log \mathrm{K}_{a}+n \log [Q], \\
F_{0} / F=a^{*} \exp \left(K_{\mathrm{sv}}{ }^{*} Q\right),
\end{gathered}
$$

where $F_{0}$ and $F$ are the fluorescence intensities in the absence and presence of ligands, respectively; $K_{a}$ and $K_{\mathrm{sv}}$ depict binding constants; $n$ is the number of the binding site(s); and $a$ is the amplitude.

For EA, a linear model as depicted in Equation (8) was used. In contrast, a nonlinear model as described by Equation (9) was applied for quercetin to fit the Stern-Volmer plot with the upward curvature having exponential dependence. This deviation from linearity (i.e., upward curvature) in the 


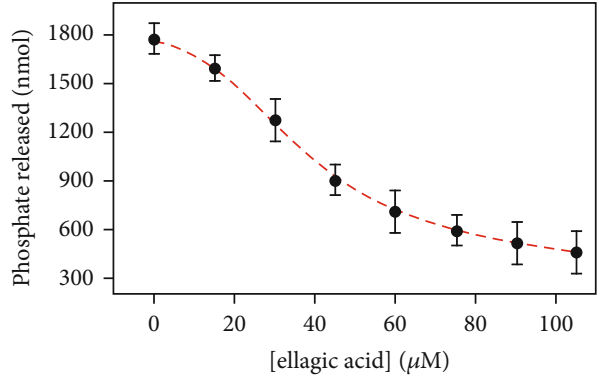

(a)



(c)

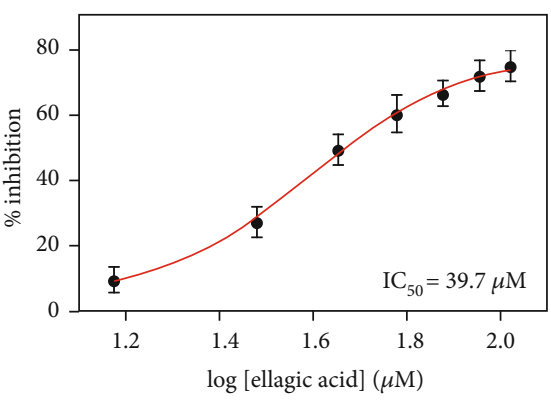

(b)

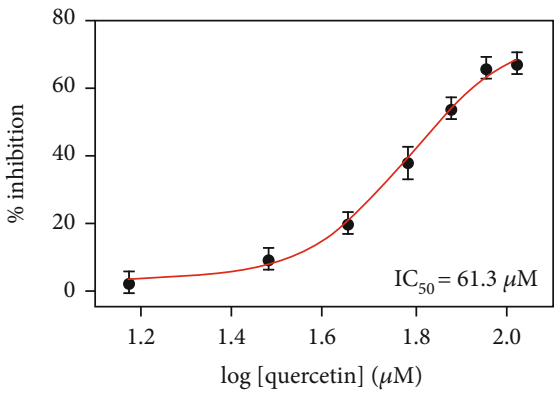

(d)

Figure 9: ATPase inhibition assay of CaMKIV with EA and quercetin. (a) Dose-response curve showing the effect of increasing concentrations of EA (0 to $100 \mu \mathrm{M})$ on the kinase activity of CaMKIV. The standard phosphate curve was used to calculate the amount of inorganic phosphate released from ATP in a reaction catalyzed by CaMKIV. (b) Plot depicting the amount of \% inhibition in ATPase activity as a function of log [EA]. The data were fit to estimate the value of IC50 for EA using GraphPad Prism 5.0. (c) The dose-response curve showing the effect of increasing concentrations of quercetin (0 to $100 \mu \mathrm{M})$ on the kinase activity of CaMKIV. (d) Plot depicting the amount of $\%$ inhibition in ATPase activity as a function of log [quercetin]. Results shown here are depicted as means \pm SD of three independent experiments.

case of quercetin can be attributed to the occurrence of both static and dynamic quenching and the differential binding of the ligand to the protein molecule. The values of binding constants obtained were $4.28 \times 10^{4} \mathrm{M}^{-1}\left(K_{\text {sv }}\right)$ and $7.82 \times 10^{2} \mathrm{M}^{-1}$ $\left(K_{\mathrm{sv}}\right)$ for EA and quercetin, respectively (Table 2$)$. The other natural compounds do not quench the intrinsic fluorescence of CAMKIV appreciably (Figure S1), and hence, they were not evaluated further in the search to identify the potent inhibitors of CaMKIV.

3.9. Enzyme Inhibition Assay. CaMKIV possess an inherent ATPase activity $[67,68]$. Thus, we have employed BIOMOL reagent-based kinase assay wherein the inorganic phosphate released by the hydrolysis of ATP in a reaction catalyzed by CaMKIV forms a green-colored complex with malachite green. The complex absorbs at $620 \mathrm{~nm}$ that gives an estimate about the catalytic activity of CaMKIV. The inhibition assay was performed in the presence of different concentrations of EA and quercetin to evaluate their capacity to inhibit the enzyme activity of CaMKIV. Figures 9(a) and 9(c) shows dose-responses curve obtained by plotting the amount of phosphate released by the ATPase activity of CaMKIV as a function of ligand concentrations. A continuous decrease in the enzyme-catalyzed ATP hydrolysis was observed in a dose-dependent manner for both EA and quercetin. Around $70 \%$ decrease in the ATPase activity of CaMKIV was noticed in the presence of EA and quercetin. The raw data were converted to $\%$ inhibition values using the formula, $100-(A)$
$\left.A_{0} \times 100\right)$ where $A_{0}$ and $A$ represent the enzyme activity of CaMKIV in the absence and presence of the compound. The percent inhibition in kinase activity was plotted against $\log$ [compound], and data were fitted to estimate the value of $\mathrm{IC}_{50}$ (50\% of ATPase inhibition) for both the compounds using GraphPad Prism 5.0 (Figures 9(b) and 9(d)). The $\mathrm{IC}_{50}$ values obtained for EA and quercetin were 39.7 and $61.3 \mu \mathrm{M}$, respectively. It is quite evident from $\mathrm{IC}_{50}$ values, that both $\mathrm{EA}$ and quercetin are potent in inhibiting the catalytic activity of CaMKIV in the micromolar range. All together, the results from fluorescence binding and enzyme activity show that EA and quercetin act as potential inhibitors of CaMKIV with appreciable binding affinity.

\section{Conclusions}

We conclude that both EA and quercetin bind to the active site pocket of CaMKIV and thus serve as potent inhibitors. They form $\mathrm{H}$-bonded interactions with active site residues, thereby significantly reduces the catalytic activity of CaMKIV. Thus, targeting CaMKIV by these phytochemicals can be a potential curative strategy for the treatment of cancer and other human diseases linked to the abnormal expression of CaMKIV. The usage of EA and quercetin as drug-like molecules can be further explored by making suitable modifications on the basic scaffold to improve their bioavailability and therapeutic potential. Overall, we believe that this study widens the scope of the utilization of natural compounds in 
the development of therapeutics for various human diseases including cancer.

\section{Data Availability}

The data used to support the findings of this study are included within the supplementary information files.

\section{Conflicts of Interest}

The authors declare no conflicts of interest.

\section{Acknowledgments}

PG thanks DST for the award of the National-Post doctoral fellowship (File no. PDF/2017/001084). MFA, MTR, and $\mathrm{AH}$ acknowledge the generous support from the Research Supporting Project (No. RSP-2020-122) by King Saud University, Riyadh, Kingdom of Saudi Arabia. FA is thankful to the Indian National Science Academy for the award of Senior Scientist Fellowship. This work is funded by the Indian Council of Medical Research (Grant No. BIC/12(01)/2015). SK and ZF would like to acknowledge the Centre for High Performance Computing (CHPC) server based in Cape Town, South Africa, for conducting the in silico work.

\section{Supplementary Materials}

Figure S1: fluorescence binding study of CaMKIV with natural compounds. Fluorescence emission spectra of CaMKIV $(4 \mu \mathrm{M})$ with increasing concentrations of compounds at pH 7.4. (a) Capsaicin. (b) Limonin. (c) Simvastatin. (d) DL- $\alpha$ tocopherol acetate. (e) Ursolic acid. Table S1: docking $g$ score of selected and screened natural compounds with their binding site residues. (Supplementary Materials)

\section{References}

[1] H. Naz, A. Islam, F. Ahmad, and M. I. Hassan, "Calcium/calmodulin-dependent protein kinase IV: a multifunctional enzyme and potential therapeutic target," Progress in Biophysics and Molecular Biology, vol. 121, no. 1, pp. 54-65, 2016.

[2] L. Racioppi and A. R. Means, "Calcium/calmodulin-dependent protein kinase kinase 2: roles in signaling and pathophysiology," The Journal of Biological Chemistry, vol. 287, no. 38, pp. 31658-31665, 2012.

[3] S. Impey, A. L. Fong, Y. Wang et al., "Phosphorylation of CBP mediates transcriptional activation by neural activity and CaM kinase IV,” Neuron, vol. 34, no. 2, pp. 235-244, 2002.

[4] Y. Sano, J. L. Shobe, M. Zhou et al., "CREB regulates memory allocation in the insular cortex," Current Biology, vol. 24, no. 23, pp. 2833-2837, 2014.

[5] M. M. Bland, R. S. Monroe, and C.-A. Ohmstede, "The cDNA sequence and characterization of the $\mathrm{Ca} 2+/$ calmodulin-dependent protein kinase-Gr from human brain and thymus," Gene, vol. 142, no. 2, pp. 191-197, 1994.

[6] S. A. Hawley, M. Davison, A. Woods et al., "Characterization of the AMP-activated protein kinase kinase from rat liver and identification of threonine 172 as the major site at which it phosphorylates AMP-activated protein kinase," Journal of Biological Chemistry, vol. 271, no. 44, pp. 27879-27887, 1996.
[7] A. R. Means, "Regulatory cascades involving calmodulindependent protein kinases," Molecular Endocrinology, vol. 14, no. 1, pp. $4-13,2000$.

[8] B. Zhivotovsky and S. Orrenius, "Calcium and cell death mechanisms: a perspective from the cell death community," Cell Calcium, vol. 50, no. 3, pp. 211-221, 2011.

[9] M. Nomura, A. Ueno, K. Saga, M. Fukuzawa, and Y. Kaneda, "Accumulation of cytosolic calcium induces necroptotic cell death in human neuroblastoma," Cancer Research, vol. 74, no. 4, pp. 1056-1066, 2014.

[10] M. M. L. Oswaldo, G. Rodriguez-Mora, M. J. Evans et al., "Inhibition of the CaM-kinases augments cell death in response to oxygen radicals and oxygen radical inducing cancer therapies in MCF-7 human breast cancer cells," Cancer Biology \& Therapy, vol. 5, no. 8, pp. 1022-1030, 2006.

[11] N. Tamura, Y. Tai, K. Sugimoto et al., "Enhanced expression and activation of $\mathrm{Ca} 2+/$ calmodulin dependent protein kinase IV in hepatocellular carcinoma," Cancer, vol. 89, no. 9, pp. 1910-1916, 2000.

[12] C. L. Williams, S. H. Phelps, and R. A. Porter, "Expression of $\mathrm{Ca} 2+/$ calmodulin-dependent protein kinase types II and IV, and reduced DNA synthesis due to the $\mathrm{Ca} 2+/$ calmodulindependent protein kinase inhibitor KN-62 (1-[N,O-bis(5-isoquinolinesulfonyl)-N-methyl-L-tyrosyl]-4-phenyl piperazine) in small cell lung carcinoma," Biochemical Pharmacology, vol. 51, no. 5, pp. 707-715, 1996.

[13] Z. Li, J. Lu, G. Zeng et al., "MiR-129-5p inhibits liver cancer growth by targeting calcium calmodulin- dependent protein kinase IV (CAMK4)," Cell Death \& Disease, vol. 10, no. 11, p. 789, 2019.

[14] L. D. McCullough, S. Tarabishy, L. Liu et al., "Inhibition of calcium/calmodulin-dependent protein kinase kinase $\beta$ and calcium/calmodulin-dependent protein kinase IV is detrimental in cerebral ischemia," Stroke, vol. 44, no. 9, pp. 2559-2566, 2013.

[15] K. Ichinose, T. Rauen, Y.-T. Juang et al., "Cutting edge: calcium/calmodulin-dependent protein kinase type IV is essential for mesangial cell proliferation and lupus nephritis," The Journal of Immunology, vol. 187, no. 11, pp. 5500-5504, 2011.

[16] P. Cohen, "Protein kinases-the major drug targets of the twenty-first century?," Nature Reviews Drug Discovery, vol. 1, no. 4, pp. 309-315, 2002.

[17] F. Naz, F. Anjum, A. Islam, F. Ahmad, and M. I. Hassan, "Microtubule affinity-regulating kinase 4: structure, function, and regulation," Cell Biochemistry and Biophysics, vol. 67, no. 2, pp. 485-499, 2013.

[18] N. Hoda, H. Naz, E. Jameel et al., "Curcumin specifically binds to the human calcium-calmodulin-dependent protein kinase IV: fluorescence and molecular dynamics simulation studies," Journal of Biomolecular Structure \& Dynamics, vol. 34, no. 3, pp. 572-584, 2015.

[19] P. Khan, A. Queen, T. Mohammad et al., "Identification of $\alpha$-mangostin as a potential inhibitor of microtubule affinity regulating kinase 4," Journal of Natural Products, vol. 82, no. 8, pp. 2252-2261, 2019.

[20] P. Khan, S. Rahman, A. Queen et al., "Elucidation of dietary polyphenolics as potential inhibitor of microtubule affinity regulating kinase 4: in silico and in vitro studies," Scientific Reports, vol. 7, no. 1, p. 9470, 2017.

[21] A. Shamsi, T. Mohammad, S. Anwar et al., "Glecaprevir and Maraviroc are high-affinity inhibitors of SARS-CoV-2 main 
protease: possible implication in COVID-19 therapy," Bioscience Reports, vol. 40, no. 6, 2020.

[22] R. Dahiya, T. Mohammad, S. Roy et al., "Investigation of inhibitory potential of quercetin to the pyruvate dehydrogenase kinase 3: towards implications in anticancer therapy," International Journal of Biological Macromolecules, vol. 136, pp. 1076-1085, 2019.

[23] P. Gupta, T. Mohammad, R. Dahiya et al., "Evaluation of binding and inhibition mechanism of dietary phytochemicals with sphingosine kinase 1: towards targeted anticancer therapy," Sci Rep, vol. 9, no. 1, p. 18727, 2019.

[24] P. Gupta, T. Mohammad, P. Khan et al., "Evaluation of ellagic acid as an inhibitor of sphingosine kinase 1: a targeted approach towards anticancer therapy," Biomedicine \& Pharmacotherapy, vol. 118, p. 109245, 2019.

[25] A. Amin, H. Gali-Muhtasib, M. Ocker, and R. SchneiderStock, "Overview of major classes of plant-derived anticancer drugs," International Journal of Biomedical Science, vol. 5, no. 1, pp. 1-11, 2009.

[26] A. N. M. Alamgir, Therapeutic Use of Medicinal Plants and their Extracts: Volume 1: Pharmacognosy, Springer, 2017.

[27] T. Mohammad, K. Arif, M. F. Alajmi et al., "Identification of high-affinity inhibitors of pyruvate dehydrogenase kinase-3: towards therapeutic management of cancer," Journal of Biomolecular Structure \& Dynamics, pp. 1-9, 2020.

[28] T. Mohammad, S. Batra, R. Dahiya et al., "Identification of high-affinity inhibitors of cyclin-dependent kinase 2 towards anticancer therapy," Molecules, vol. 24, no. 24, p. 4589, 2019.

[29] M. Gulzar, S. Ali, F. I. Khan, P. Khan, P. Taneja, and M. I. Hassan, "Binding mechanism of caffeic acid and simvastatin to the integrin linked kinase for therapeutic implications: a comparative docking and MD simulation studies," Journal of Biomolecular Structure \& Dynamics, vol. 37, no. 16, pp. 4327-4337, 2018.

[30] M. Gulzar, S. B. Syed, F. I. Khan et al., "Elucidation of interaction mechanism of ellagic acid to the integrin linked kinase," International Journal of Biological Macromolecules, vol. 122, pp. 1297-1304, 2019.

[31] J. Grein, N. Ohmagari, D. Shin et al., "Compassionate use of remdesivir for patients with severe Covid-19," New England Journal of Medicine, vol. 382, no. 24, pp. 23272336, 2020.

[32] H. Naz, P. Khan, M. Tarique et al., "Binding studies and biological evaluation of $\beta$-carotene as a potential inhibitor of human calcium/calmodulin-dependent protein kinase IV," International Journal of Biological Macromolecules, vol. 96, pp. 161-170, 2017.

[33] H. Naz, M. Tarique, P. Khan et al., "Evidence of vanillin binding to CAMKIV explains the anti-cancer mechanism in human hepatic carcinoma and neuroblastoma cells," Molecular and Cellular Biochemistry, vol. 438, no. 1-2, pp. 35-45, 2018.

[34] A. Beg, F. I. Khan, K. A. Lobb, A. Islam, F. Ahmad, and M. I. Hassan, "High throughput screening, docking, and molecular dynamics studies to identify potential inhibitors of human calcium/calmodulin-dependent protein kinase IV," Journal of Biomolecular Structure \& Dynamics, vol. 37, no. 8, pp. 21792192, 2018.

[35] S. Schrödinger Release 2020-1: Protein Preparation Wizard:Epik, LLC, New York, NY, 2016.

[36] Impact, Schrödinger, LLC, New York, NY, 2016.

[37] Prime, Schrödinger, LLC, New York, NY, 2020.
[38] E. Harder, W. Damm, J. Maple et al., “OPLS3: a force field providing broad coverage of drug-like small molecules and proteins," Journal of Chemical Theory and Computation, vol. 12, no. 1, pp. 281-296, 2015.

[39] S. Schrödinger Release 2020-1: LigPrep, LLC, New York, NY, 2020.

[40] T. A. Halgren, R. B. Murphy, R. A. Friesner et al., "Glide: a new approach for rapid, accurate docking and scoring. 2. Enrichment factors in database screening," Journal of Medicinal Chemistry, vol. 47, no. 7, pp. 1750-1759, 2004.

[41] I. Y. B.-S. D. A. Case, S. R. Brozell, D. S. Cerutti et al., AMBER 2018, University of California, San Francisco, 2018.

[42] P. Robustelli, S. Piana, and D. E. Shaw, "Developing a molecular dynamics force field for both folded and disordered protein states," Proceedings of the National Academy of Sciences, vol. 115, no. 21, pp. E4758-E4766, 2018.

[43] J. Wang, R. M. Wolf, J. W. Caldwell, P. A. Kollman, and D. A. Case, "Development and testing of a general amber force field," Journal of Computational Chemistry, vol. 25, no. 9, pp. 1157-1174, 2004.

[44] J. Wang, W. Wang, P. A. Kollman, and D. A. Case, “Automatic atom type and bond type perception in molecular mechanical calculations," Journal of Molecular Graphics and Modelling, vol. 25, no. 2, pp. 247-260, 2006.

[45] A. W. Götz, M. J. Williamson, D. Xu, D. Poole, S. le Grand, and R. C. Walker, "Routine microsecond molecular dynamics simulations with AMBER on GPUs. 1. Generalized born," Journal of Chemical Theory and Computation, vol. 8, no. 5, pp. 15421555, 2012.

[46] D. R. Roe and T. E. Cheatham, "PTRAJ and CPPTRAJ: software for processing and analysis of molecular dynamics trajectory data," Journal of Chemical Theory and Computation, vol. 9, no. 7, pp. 3084-3095, 2013.

[47] P. K. Janert, Gnuplot in Action: Understanding Data with Graphs, Manning Publications Co., 2009.

[48] E. Wang, H. Sun, J. Wang et al., "End-point binding free energy calculation with MM/PBSA and MM/GBSA: strategies and applications in drug design," Chemical Reviews, vol. 119, no. 16, pp. 9478-9508, 2019.

[49] H. Naz, M. Shahbaaz, M. A. Haque et al., "Urea-induced denaturation of human calcium/calmodulin-dependent protein kinase IV: a combined spectroscopic and MD simulation studies," Journal of Biomolecular Structure \& Dynamics, vol. 35, no. 3, pp. 463-475, 2016.

[50] B. Aneja, N. S. Khan, P. Khan et al., "Design and development of isatin-triazole hydrazones as potential inhibitors of microtubule affinity-regulating kinase 4 for the therapeutic management of cell proliferation and metastasis," European Journal of Medicinal Chemistry, vol. 163, pp. 840-852, 2019.

[51] B. Aneja, A. Queen, P. Khan et al., "Design, synthesis \& biological evaluation of ferulic acid-based small molecule inhibitors against tumor-associated carbonic anhydrase IX," Bioorganic \& Medicinal Chemistry, vol. 28, no. 9, article 115424, 2020.

[52] P. Gupta, F. I. Khan, S. Roy et al., "Functional implications of $\mathrm{pH}$-induced conformational changes in the sphingosine kinase 1," Spectrochimica Acta. Part A, Molecular and Biomolecular Spectroscopy, vol. 225, p. 117453, 2020.

[53] S. Muthukumaran, C. Tranchant, J. Shi, X. Ye, and S. J. Xue, "Ellagic acid in strawberry (Fragaria spp.): biological, technological, stability, and human health aspects," Food Quality and Safety, vol. 1, no. 4, pp. 227-252, 2017. 
[54] F. I. Khan, M. Aamir, D.-Q. Wei, F. Ahmad, and M. I. Hassan, "Molecular mechanism of Ras-related protein Rab-5A and effect of mutations in the catalytically active phosphatebinding loop," Journal of Biomolecular Structure and Dynamics, vol. 35, no. 1, pp. 105-118, 2016.

[55] S. Khan, I. Bjij, and M. E. S. Soliman, "Selective covalent inhibition of "allosteric Cys121" distort the binding of PTP1B enzyme: a novel therapeutic approach for cancer treatment," Cell Biochemistry and Biophysics, vol. 77, no. 3, pp. 203-211, 2019.

[56] F. Naz, F. I. Khan, T. Mohammad et al., "Investigation of molecular mechanism of recognition between citral and MARK4: a newer therapeutic approach to attenuate cancer cell progression," International Journal of Biological Macromolecules, vol. 107, Part B, pp. 2580-2589, 2018.

[57] A. Kuzmanic and B. Zagrovic, "Determination of ensembleaverage pairwise root mean-square deviation from experimental B-factors," Biophysical Journal, vol. 98, no. 5, pp. 861-871, 2010.

[58] S. Ali, F. Khan, T. Mohammad, D. Lan, M. Hassan, and Y. Wang, "Identification and evaluation of inhibitors of lipase from Malassezia restricta using virtual high-throughput screening and molecular dynamics studies," International Journal of Molecular Sciences, vol. 20, no. 4, p. 884, 2019.

[59] T. Mohammad, F. I. Khan, K. A. Lobb, A. Islam, F. Ahmad, and M. I. Hassan, "Identification and evaluation of bioactive natural products as potential inhibitors of human microtubule affinity-regulating kinase 4 (MARK4)," Journal of Biomolecular Structure and Dynamics, vol. 37, no. 7, pp. 1813-1829, 2018.

[60] S. Khan, I. Bjij, R. M. Betz, and M. E. S. Soliman, "Reversible versus irreversible inhibition modes of ERK2: a comparative analysis for ERK2 protein kinase in cancer therapy," Future Medicinal Chemistry, vol. 10, no. 9, pp. 1003-1015, 2018.

[61] S. Ali, M. Hassan, A. Islam, and F. Ahmad, "A review of methods available to estimate solvent-accessible surface areas of soluble proteins in the folded and unfolded states," Current Protein \& Peptide Science, vol. 15, no. 5, pp. 456-476, 2014.

[62] H. Chen and A. Z. Panagiotopoulos, "Molecular modeling of surfactant micellization using solvent-accessible surface area," Langmuir, vol. 35, no. 6, pp. 2443-2450, 2019.

[63] E. Jameel, H. Naz, P. Khan et al., "Design, synthesis, and biological evaluation of pyrimidine derivatives as potential inhibitors of human calcium/calmodulin-dependent protein kinase IV," Chemical Biology \& Drug Design, vol. 89, no. 5, pp. 741-754, 2017.

[64] H. Naz, E. Jameel, N. Hoda et al., "Structure guided design of potential inhibitors of human calcium-calmodulin dependent protein kinase IV containing pyrimidine scaffold," Bioorganic \& Medicinal Chemistry Letters, vol. 26, no. 3, pp. 782-788, 2016.

[65] J. R. Lakowicz, "Quenching of fluorescence," in Principles of Fluorescence Spectroscopy,, pp. 277-330, Springer US, 2006.

[66] J. Carvalho, P. Nottelet, J.-L. Mergny, J. A. Queiroz, G. F. Salgado, and C. Cruz, "Study of the interaction between indole-based compounds and biologically relevant G-quadruplexes," Biochimie, vol. 135, pp. 186-195, 2017.
[67] H. Naz, M. Tarique, S. Ahamad et al., "Hesperidin-CAMKIV interaction and its impact on cell proliferation and apoptosis in the human hepatic carcinoma and neuroblastoma cells," Journal of Cellular Biochemistry, vol. 120, no. 9, pp. 1511915130, 2019.

[68] H. Naz, M. Shahbaaz, K. Bisetty, A. Islam, F. Ahmad, and M. I. Hassan, "Effect of $\mathrm{pH}$ on the structure, function, and stability of human calcium/calmodulin-dependent protein kinase IV: combined spectroscopic and MD simulation studies," Biochemistry and Cell Biology, vol. 94, no. 3, pp. 221-228, 2016. 